\title{
Sampling Ocean Materials, Traces of Life or Biosignatures in Plume Deposits on Enceladus' Surface
}

A Science White Paper for the Planetary Science and Astrobiology Decadal Survey for 2023-2032

Choukroun M. ${ }^{1}$, Backes P. ${ }^{\text {I }}$, Cable M.L. ${ }^{1}$, Hodyss R. ${ }^{l}$, Badescu M. ${ }^{\text {I }}$, Marteau E. ${ }^{1}$, Molaro J.L. ${ }^{2}$, Moreland S. ${ }^{1}$, Nordheim T.A. ${ }^{1}$, Okamoto T. ${ }^{1}$, Riccobono D. ${ }^{3}$, Zacny K. ${ }^{4}$

1: Jet Propulsion Laboratory, California Institute of Technology, 4800 Oak Grove Dr., MS 183-

601, Pasadena CA 91109 (phone: +1 (818) 354-4875, E-mail: mathieu.choukroun@jpl.nasa.gov)

2: Planetary Science Institute, Tucson, AZ.

3: Politecnino di Torino, Torino, Italy.

4: Honeybee Robotics, Pasadena, CA.

\begin{abstract}
Plume deposit regions on the surface of Enceladus likely reflect and preserve the composition of the internal ocean and could contain potential traces of life and biosignatures. The novel DualRasp sampling system is designed to acquire large amounts of plume deposits on the surface of Enceladus. It is under development to achieve TRL 5 in 2021.
\end{abstract}

To endorse this white paper and add your name to the list of co-signatories, please visit: https://docs.google.com/forms/d/e/1FAIpQLSfomWb0C87qG$\underline{\text { PAEW80qP9RMaOf1N9fq2i37W7DtlyuY6ckVg/viewform }}$

Co-Signatories: https://docs.google.com/document/d/1YeHphpEVk8bf0yPj82464hfEQVnd9G2aqTO2OKrKxI/edit 
Enceladus is unique as an astrobiology target in that it hosts an active plume sourced directly from its habitable subsurface ocean. Ice particles from the plume contain geochemical constituents that are diagnostic of the ocean conditions, and may hold traces of life and/or biosignatures as well, if they exist.

Up to $93 \%$ of the plume particles fall back onto the surface of Enceladus. The low radiation environment (compared to Europa) and present-day activity are favorable to the preservation of any complex organics and putative biosignatures contained within these particles. Laboratory experiments and modeling suggest that plume deposits would likely be poorly consolidated and relatively easy to sample.

Sampling systems like a dual rasp, under development to achieve TRL 5 in 2021, would enable a landed mission on Enceladus' surface to acquire large amounts of surface materials, a requirement for analysis of trace constituents. A landed mission on Enceladus could further our understanding of the chemical make-up of plume particles and the subsurface ocean, and seek traces of life and/or biosignatures.

\section{Enceladus emits materials from a potentially habitable interior ocean, and these materials are largely re-deposited on the surface}

Enceladus is, to our knowledge, the only Ocean World where material from its habitable, subsurface ocean is being actively ejected into space and onto its surface. Cassini gravity data, in conjunction with surface images showing a libration ('wobble') [1], indicate that Enceladus contains a global ocean beneath its ice shell [2]. Cassini observed over a hundred jets converging into a plume that originates from the South polar terrain of Enceladus [3], and particularly from a set of four rectilinear surface fractures dubbed Tiger Stripes [4]. The Tiger Stripes exhibit local thermal anomalies [5, 6] with a temperature up to $180 \mathrm{~K}$ [7], while the rest of the South pole terrain is much colder, around 30-50 K [8]. Intense tidal dissipation within the ice shell is likely responsible for the thermal anomalies and the existence of the plume [9, 10]. Evidence from Voyager observations and modeling from Cassini data suggest the plume is a long-lived phenomenon, persisting for decades and perhaps even longer [11-13].

Enceladus' plume consists of vapor and particles; the particles are approximately micronsized and mostly comprised of water ice and feed Saturn's E ring [14]. A subset ( $40 \%)$ of the particles also contain percent-level $\mathrm{NaCl}$ and other salts by mass $[15,16]$ and a separate subset ( $\sim 4 \%$ ) contains complex organic materials, also at the percent-level by mass [17]. The vapor phase of the plume includes ammonia, carbon dioxide, low-mass organics including $\mathrm{CH}_{4},{ }^{40} \mathrm{Ar}$ [18], and molecular $\mathrm{H}_{2}$ [19]. ${ }^{40} \mathrm{Ar}$ is formed from the radioactive decay of ${ }^{40} \mathrm{~K}$, which suggests a direct connection between the silicate interior and the exosphere. $\mathrm{H}_{2}$ and $\mathrm{CH}_{4}$ are strongly suggestive of ongoing hydrothermal activity, as it would leave the interior and escape in a short period of time. The moderately high $\mathrm{pH}$ derived for the ocean [20], the presence of complex organic materials, and the abundant geothermal energy from the interior and within the South polar terrain provide evidence for habitability and the enticing prospect that life may have emerged and still be present on Enceladus [21-23].

Cassini observations of particles in the Enceladus plume and the E ring enabled the determination of their grain size distribution and their trajectories, and the modeling of their 
deposition rate back on Enceladus surface. The mean radius of equivalent-sphere particles determined from imaging is $3.1 \pm 0.5 \mu \mathrm{m}$ [24]. The Cassini Cosmic Dust Analyzer characterized the vertical structure of the plume, from which a particle ejection model was established [25]. The deposition of plume particles could then be computed as function of particle size, source location, and location on the Enceladus surface $[14,26]$. Particles in the range $0.1-5 \mu \mathrm{m}$ are expected to dominate the plume deposits. The average deposition rate is on order of $1 \mu \mathrm{m} / \mathrm{yr}$, but can exceed $0.1 \mathrm{~mm} / \mathrm{yr}$ in locations close to jet sources $[14,26]$. This is roughly consistent with a separate model that suggests $68-93 \%$ of all plume particles are deposited on the surface [27].

Plume particles, in Enceladus' exosphere or on its surface, could contain traces of life or biosignatures [27-29]. Mission concepts have previously proposed to capture particles in the plume itself [30, 31]. Landed mission concepts, in some ways similar to the Europa Lander [32], would have their own challenges and foreseeably be costly, however they would avoid hypervelocity capture from potentially affecting the mass spectral signature of plume materials. Landed mission concepts could be able to acquire much larger amounts of materials than plume fly-through concepts, allowing higher sensitivity to trace constituents, and enabling the conduction of a larger number of replicate analyses, thereby providing greater robustness of science results. For a detailed report on a potential flagship-class lander to address life detection and other questions at Enceladus, see the Planetary Mission Concept Study entitled "The Enceladus Orbilander: A Flagship-class Mission Concept for Astrobiology" by MacKenzie et al., 2020. Such mission concepts typically include multiple, independent tests for life, following the 'Ladder of Life' detection strategy [33].

\section{Traces of life and/or biosignatures could be preserved in plume deposits}

In comparison to Europa, the surface environment of Enceladus is benign, enabling preservation of organic molecules on the surface for relatively long timescales. Both particle and ultraviolet radiation can have degradative effects on biosignatures. Uniquely on Enceladus, these effects are modulated by the continuing deposition of fresh plume particles, which scatter or absorb radiation and shield buried particles.

The flux of magnetospheric particle radiation at Enceladus is relatively low. While studies on the particle irradiation at Enceladus are not available, we can use the radiation environment of Saturn's moon Mimas as a worst-case analog for Enceladus. On the most irradiated location on Mimas, the time to reach $100 \mathrm{ev} / 16$ amu electron dose accumulation (a standard unit representing a chemically significant dose) is about 1 million years at a depth of $1 \mathrm{~mm}$ [34]. Thus, shallow material in regions of plume deposition will be minimally processed. Galactic cosmic rays (GCRs) deliver radiation doses that are many orders of magnitude lower than that from magnetospheric particles, thus do not need to be considered as a degradation mechanism on the short timescales appropriate to Enceladus' continually deposited plume particles.

Solar ultraviolet irradiation can also degrade organic biosignatures. Generally, the most damaging radiation is in the vacuum ultraviolet (VUV), below approximately $150 \mathrm{~nm}$. Light at these wavelengths and below has absorption lengths (the distance light travels before its intensity decreases buy a factor of 1/e) in single crystal water ice of less than $0.1 \mu \mathrm{m}$ [35]. This indicates that organic molecules embedded within micron-sized plume grains will be almost entirely shielded from damaging VUV radiation. Continuing deposition on the surface would provide additional shielding. 
Longer wavelength ultraviolet radiation is also damaging to organic molecules. For example, the photolytic half-lives of the amino acids glycine and phenylalanine are 6.5 and 4.5 years at $206 \mathrm{~nm}$, and 5 and 1 years at $254 \mathrm{~nm}$, respectively, under Solar flux levels representative of Europa's surface [36]. Note that the Solar flux at Enceladus would be 3.3 times less than at Europa. Absorption lengths for photons at these wavelengths are approximately $1 \mathrm{~m}$ in a pure block of water ice. However, scattering models [36] show penetration depths of 250 and $500 \mu \mathrm{m}$ at 210 and $300 \mathrm{~nm}$ in a non-porous medium of isotropically scattering $60 \mu \mathrm{m}$ ice grains.

Scattering, and thus penetration depths, would be even shorter in a medium composed of smaller particles, as expected at Enceladus. We conclude that at locations on Enceladus where deposition rates are high $(0.1 \mathrm{~mm} /$ year and higher $)$, the deposition of plume particles would effectively shield amino acids from photolytic degradation. We expect that most other organic molecular biosignatures would have photolytic half-lives comparable to amino acids.

\section{Plume deposit regions on Enceladus' surface would likely be poorly consolidated, even extensive periods of time after their deposition}

Plume deposits are expected to consist initially of fine-grained ice particles loosely in contact, forming a granular and non-cohesive material. This material would then slowly sinter over time and presumably become more consolidated $[37,38]$. The mechanical properties of plume deposits and how they evolve over time are at present not well constrained.

A recent laboratory study [39] investigated the evolution in strength upon sintering of finegrained ice particles with diameters comparable to Enceladus' plume particles. Cone penetration resistance measurements were obtained as a function of time and at different temperatures, Figure 1-a. An Arrhenius analysis of the strengthening rates yielded an activation energy of 24.3 $\mathrm{kJ} / \mathrm{mol}$, which was then used to predict the strength evolution of plume deposits under Enceladus and Europa's surface conditions, Figure 1-b.
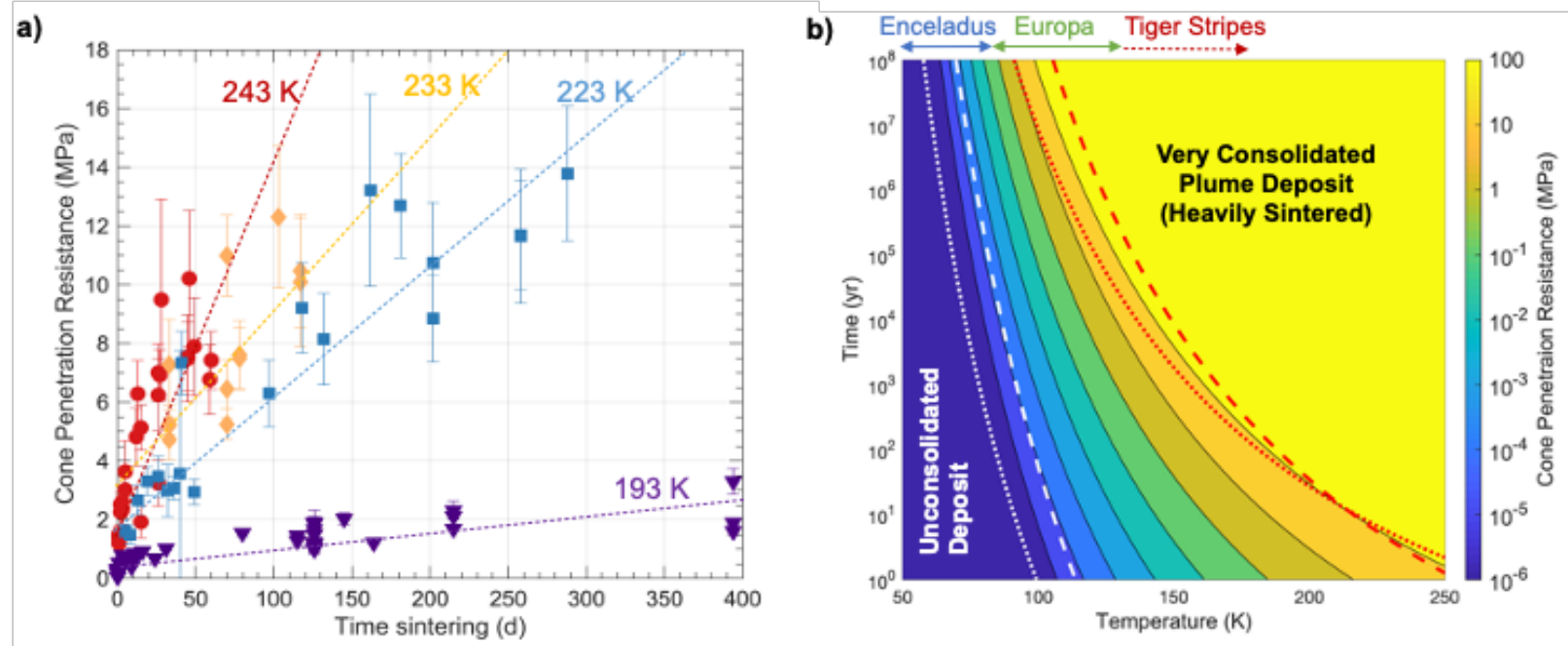

Figure 1. a) Evolution with time of the cone penetration resistance of ice plume deposit analogs at several temperatures. b) Predicted cone penetration resistance of icy plume deposits as a function of temperature and sintering time. Black contours are for the best-fit activation energy value. Dashed and dotted contours illustrate the effect of the uncertainty on activation energy. For legibility, these contours are only shown for the $10^{-5}$ (white) and 100 $\mathrm{MPa}$ (red) cone penetration resistance levels. Modified after Choukroun et al. [39]. 
Based on these results, plume deposits on Enceladus are expected to be poorly consolidated. It would take at least $100 \mathrm{My}$ from plume deposits to develop a resistance of $1 \mathrm{MPa}$ under Enceladus's nominal surface conditions. Deposits near the Tiger Stripes, where strengthening rates would be much higher, would also be covered by fresh unconsolidated particles at a rate up to $\sim 1 \mathrm{~mm} / \mathrm{yr}[14,26]$.

Plume deposits on Enceladus would likely be poorly consolidated, thus low-force sampling systems are anticipated to be able to acquire large volumes of materials in all areas of Enceladus where these materials are abundant.

\section{A dual rasp sampling system can acquire samples from plume deposit regions}

An Enceladus lander science requirement of shallowest material acquired from a potential wide range of weak to strong icy material in the low 1\% Earth gravity vacuum environment results in a unique challenge for sampling. Prior sampling systems were designed for different applications and requirements that make them less suited to the Enceladus environment. The OSIRIS-REx mission's TAGSAM (Touch-And-Go Sample Acquisition Mechanism) was developed for the loose surface material of an asteroid [40]. The Pneumatic Sampler for the JAXA MMX (Martian Moons eXploration) mission is designed for the loose surface of Phobos [41]. The Rosetta mission's Philae lander rotary drill, SD2 (Sampler, Drill and Distribution), was designed to acquire a subsurface comet sample [42]. The Dragonfly mission's DrACO (Drill for Acquisition of Complex Organics) sampler was designed for sampling in the relatively high gravity and high atmospheric pressure environment of Titan [43]. The Mars Phoenix ISAD (Icy Soil Acquisition Device) scoop with rasp was designed for the relatively high Mars gravity environment [44].

The Dual-Rasp sampling system uniquely provides the capabilities needed to acquire the surface samples for an Enceladus landed mission [45]. It is desired that a sampling system would acquire only very shallow surface material in the top $\sim 1 \mathrm{~cm}$ since this would be the freshest plume material that has fallen to the surface. Fresh material is less likely to have been altered by UV radiation, impact gardening, or other processes, and is more representative of the current state of Enceladus' subsurface ocean. Given the expected plume deposit strength of $<1 \mathrm{MPa}$ [39], and allowing for strength uncertainty and need for mission robustness, the sampling system was developed [45] to be able to sample from surface material up to $\sim 10 \mathrm{MPa}$ CPT (cone penetration test) strength. The low 1\% Earth gravity environment at Enceladus limits the allowable reacted load from the sampling activity to a lander to less than $8 \mathrm{~N}$ [46]. Rasps are able to sample the stronger material while imparting less than $8 \mathrm{~N}$ reacted load [44, 46], while other sampling tools such as drills and scoops require higher reacted loads, even to acquire samples from a $1 \mathrm{MPa}$ CPT strength material.

The Dual-Rasp sampling system has been developed to TRL 4 (Figure 2) and is under development to achieve TRL 5 in 2021. The sampler has counter-rotating rasps that break apart surface material and throw it up between them for capture in a collection cup, see Figure 4. Rasps are able to acquire a wide range of weak to strong materials with a low sampling preload. 


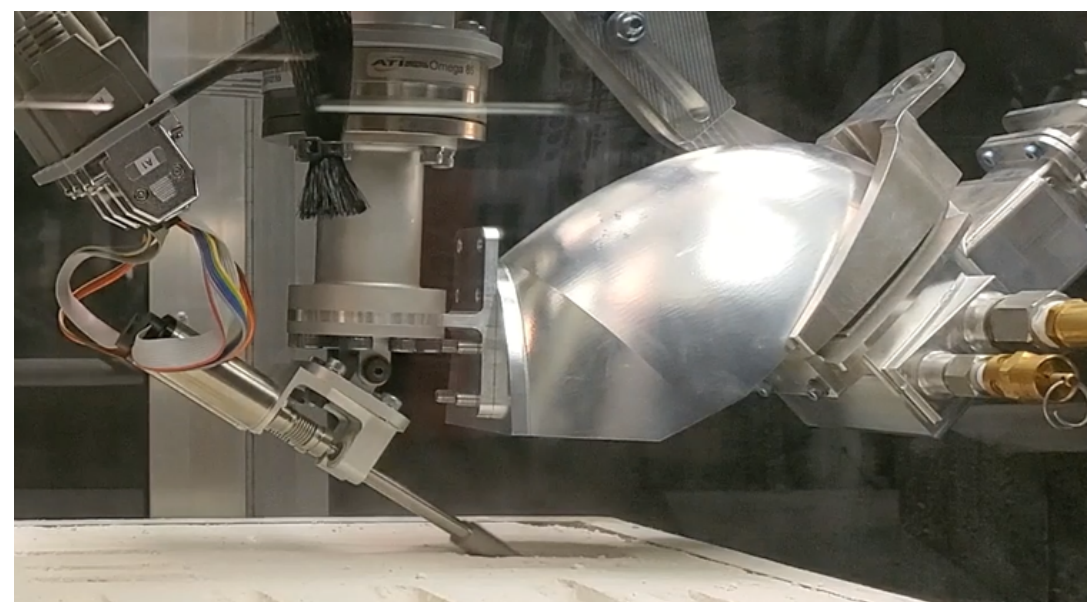

Figure 2. The Dual-Rasp sampling system is being developed to uniquely provide the sampling capabilities needed for a landed Enceladus mission.

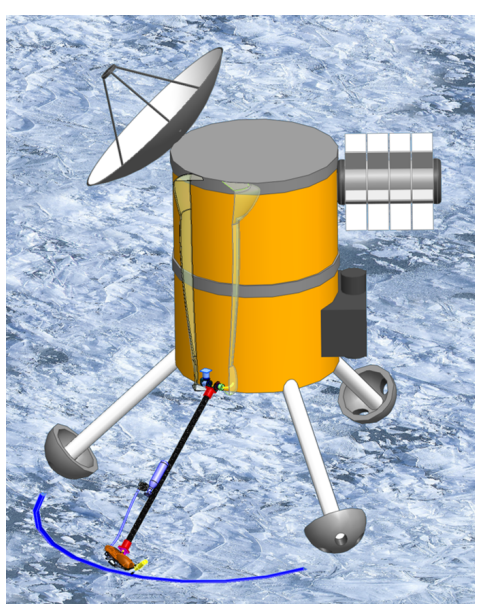

Figure 3. Sampler deployed and sampling across an arc.

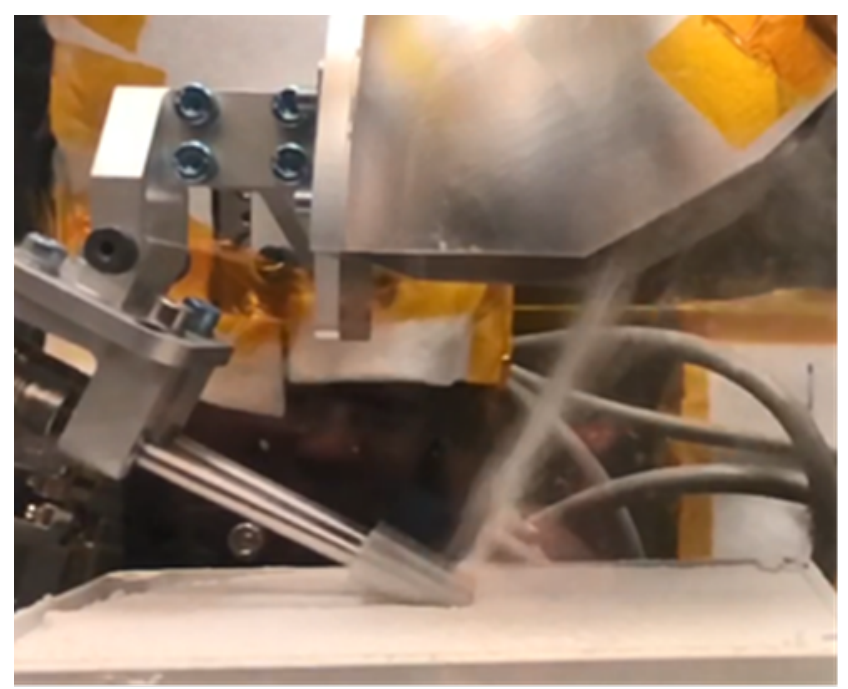

Figure 4. Counter-rotating rasps acquire and throw the sample up between them into the guide.

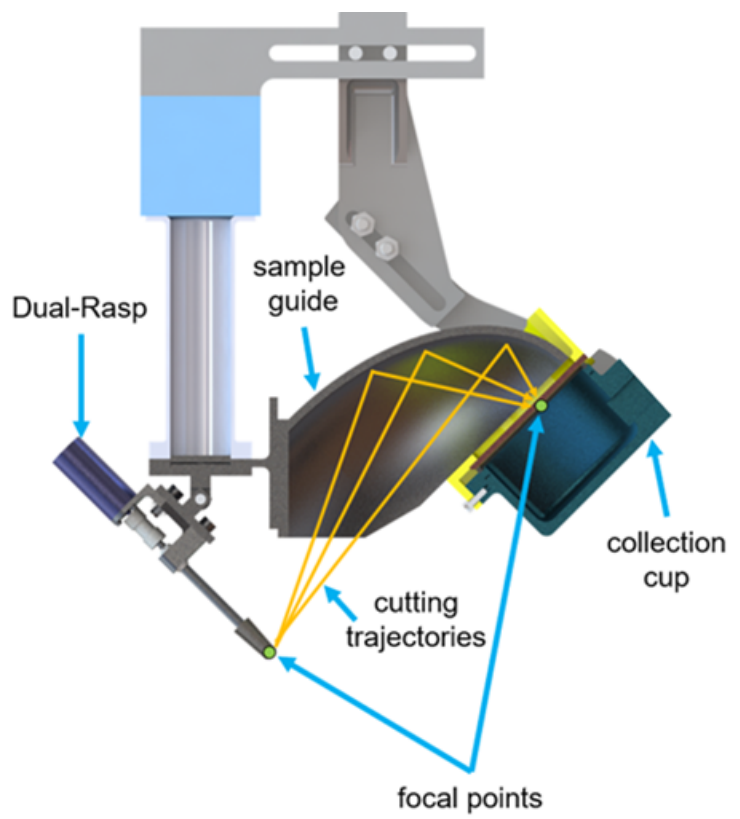

Figure 5. The sample guide elliptical shape directs the sample stream into the sample collection cup.

The Dual-Rasp is anticipated to be deployed from a lander by a two degree-of-freedom robotic arm which allows for sampling across an arc in front of the lander (Figure 3) while enabling pneumatic sample transfer through rigid pneumatic tubes. Cuttings created by the rasps and thrown into a guide are directed into the sample collection cup. The guide is shaped as an ellipsoid with the rasps at one focus and the second focus at the entrance of the sample collection cup, see Figure 5. A grid pattern at the entrance of the sample cup, not shown, prevents the sample particles from bouncing out of the sample cup. After sample has accumulated in the sample cup, the cup would be covered and compressed gas from a tank on the deployment arm would lift and transfer the sample from the collection cup, through a rigid tube up the deployment arm, through passive pneumatic joints at the arm base, and to a sample handling system for science instruments on the lander. 


\section{Summary}

Plume deposit regions on the surface of Enceladus could provide an opportunity for planetary missions to investigate the composition of another solar system ocean and determine if life exists independent of Earth. The plume deposits likely reflect and preserve the composition of the internal ocean and could contain potential traces of life and biosignatures, if they exist.

Laboratory experiments can provide the potential range of surface mechanical properties. The Enceladus surface environment presents unique challenges for sampling including low gravity, vacuum, cryogenic temperatures, and science preference for very shallow (and therefore young) surface samples. The novel Dual-Rasp sampling system uniquely provides the capabilities necessary to acquire the required surface samples. It is under development to achieve TRL 5 in 2021 to make it available for an Enceladus landed mission in the next decade.

\section{Acknowledgements}

Part of this work has been conducted at the Jet Propulsion Laboratory, California Institute of Technology, under contract to the National Aeronautics and Space Administration. Support from the JPL Research and Technology Development Program is gratefully acknowledged. Copyright 2020. All rights reserved. Government sponsorship acknowledged.

\section{References}

1. Thomas, P., et al. (2016). Icarus, 264: p. 37-47.

2. $\quad$ Iess, L., et al. (2014). Science, 344(6179): p. 78-80.

3. Porco, C.C., et al. (2006). Science, 311(5766): p. 1393-1401.

4. Spitale, J.N. and C.C. Porco (2007). Nature, 449(7163): p. 695-697.

5. Howett, C., et al. (2011). Journal of Geophysical Research: Planets, 116(E3).

6. $\quad$ Spencer, J., et al. (2006). Science, 311(5766): p. 1401-1405.

7. Spencer, J.R., et al., Enceladus: An active cryovolcanic satellite, in Saturn from CassiniHuygens. 2009, Springer. p. 683-724.

8. Howett, C., et al. (2010). Icarus, 206(2): p. 573-593.

9. Nimmo, F., et al. (2007). Nature, 447(7142): p. 289-291.

10. Spencer, J.R. and F. Nimmo (2013). Annual Review of Earth and Planetary Sciences, 41: p. 693-717.

11. Haff, P., A. Eviatar, and G. Siscoe (1983). Icarus, 56(3): p. 426-438.

12. Hemingway, D.J., M.L. Rudolph, and M. Manga (2020). Nature Astronomy, 4(3): p. 234-239.

13. Lainey, V., et al. (2020). Nature Astronomy: p. 1-6.

14. Kempf, S., U. Beckmann, and J. Schmidt (2010). Icarus, 206(2): p. 446-457.

15. Postberg, F., et al. (2009). Nature, 459(7250): p. 1098-1101.

16. Postberg, F., et al. (2011). Nature, 474(7353): p. 620-622.

17. Postberg, F., et al. (2018). Nature, 558(7711): p. 564-568.

18. Waite, J.H., et al. (2009). Nature, 460(7254): p. 487.

19. Waite, J.H., et al. (2017). Science, 356(6334): p. 155-159.

20. Glein, C.R. and J.H. Waite (2020). Geophysical Research Letters, 47(3): p. e2019GL085885.

21. McKay, C., et al. (2018). Enceladus and the Icy Moons of Saturn; Schenk, PM, Clark, RN, Howett, CJA, Verbiscer, AJ, Waite, JH, Eds: p. 437-452.

22. McKay, C.P., et al. (2014). Astrobiology, 14(4): p. 352-355. 
23. McKay, C.P., et al. (2008). Astrobiology, 8(5): p. 909-919.

24. Ingersoll, A.P. and S.P. Ewald (2011). Icarus, 216(2): p. 492-506.

25. Schmidt, J., et al. (2008). Nature, 451: p. 685.

26. Southworth, B.S., S. Kempf, and J. Spitale (2019). Icarus, 319: p. 33.

27. Porco, C.C., L. Dones, and C. Mitchell (2017). Astrobiology, 17(9): p. 876-901.

28. Guzman, M., et al. (2019). International Journal of Astrobiology, 18(1): p. 47-59.

29. Cable, M.L., et al., Enceladus, in Planetary Astrobiology, e.a. V. Meadows, Editor. 2020, University of Arizona Press and Lunar and Planetary Institute. p. in press.

30. Cable, M.L., et al. Enceladus Life Finder: The search for life in a habitable moon. in IEEE Aerospace Conference. 2016. Big Sky, MT.

31. Eigenbrode, J., et al. Searching for Life in an Ocean World: The Enceladus Life Signatures and Habitability (ELSAH) mission concept. in 42nd COSPAR Scientific Assembly. 2018.

32. Hand, K.P., Report of the Europa Lander science definition team. 2017: National Aeronautics and Space Administration.

33. Neveu, M., et al. (2018). Astrobiology, 18(11): p. 1375-1402.

34. Nordheim, T., et al. (2017). Icarus, 286: p. 56-68.

35. Warren, S.G. (1984). Applied optics, 23(8): p. 1206-1225.

36. Johnson, P.V., et al. (2012). Icarus, 221(2): p. 800-805.

37. Blackford, J.R. (2007). Journal of Physics D Applied Physics, 40: p. R355.

38. Molaro, J.L., et al. (2019). Journal of Geophysical Research (Planets), 124: p. 243-277.

39. Choukroun, M., et al. (in press). Geophysical Research Letters.

40. Beshore, E., et al. The OSIRIS-REx asteroid sample return mission. in 2015 IEEE Aerospace Conference. 2015.

41. Zacny, K., et al. Pneumatic Sampler (P-Sampler) for the Martian Moons eXploration (MMX) Mission. in IEEE Aerospace Conference. 2020.

42. Finzi, A.E., et al. (2007). Space science reviews, 128(1-4): p. 281-299.

43. Zacny, K., et al., Drill for Acquisition of Complex Organics (DrACO) for Dragonfly, a New Frontiers Mission to Explore Titan, in Lunar and Planetary Science Conference. 2020. p. 1763.

44. Bonitz, R.G., et al. (2008). Journal of Geophysical Research: Planets, 113(E3).

45. Backes, P., et al. The Dual-Rasp Sampling System for an Enceladus Lander. in IEEE Aerospace Conference. 2020.

46. Badescu, M., et al. Sampling Tool Concepts for Enceladus Lander In-Situ Analysis. in 2019 IEEE Aerospace Conference. 2019. 